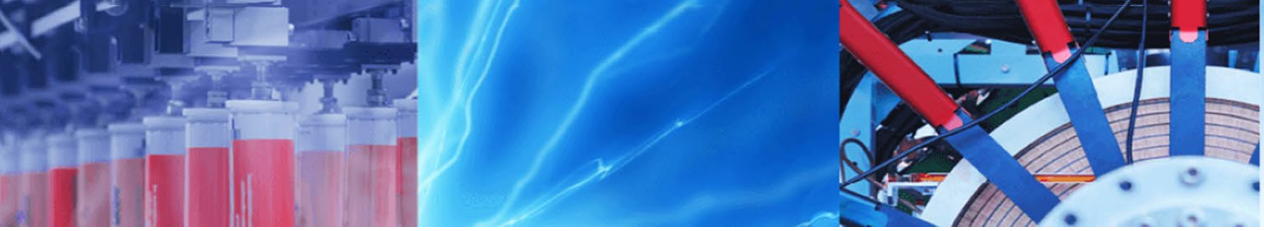

Research Article

\title{
Validation of $L C$ method in determining enantiomeric purity of pramipexole using polysaccharide-derived chiral stationary phases under organic-aqueous mode
}

\author{
Ching-Chia Chang ${ }^{1} \cdot$ Shu-Yu Fan ${ }^{1} \cdot$ Chi-Zong Zang ${ }^{1} \cdot$ Ya-Hui Hsu ${ }^{1} \cdot$ Mei-Chih Lin ${ }^{1} \cdot$ Der-Yuan Wang ${ }^{1}$. \\ Hwei-Fang Cheng ${ }^{1}$
}

(c) Springer Nature Switzerland AG 2018

\begin{abstract}
The separation of pramipexole enantiomers using polysaccharide-based chiral stationary phase (CSP) columns with organic-aqueous mobile phases was reported for the first time. The $(R)$-enantiomer was separated from pramipexole using a Lux Cellulose- 1 column with a mobile phase consisting of solvent $A$ (acetonitrile):solvent $B(0.1 \%$ diethylamine with $20 \mathrm{mM}$ ammonium bicarbonate) (30:70, v/v). The elution time was about $7.0 \mathrm{~min}$ and the resolution between the enantiomers was greater than 8.0. The linear range of the method was $0.3-3.6 \mu \mathrm{g} \mathrm{mL}^{-1}$ with the limit of detection and limit of quantification for the $(R)$-enantiomer were $0.1 \mu \mathrm{g} \mathrm{mL}^{-1}$ and $0.3 \mu \mathrm{g} \mathrm{mL}^{-1}$, respectively. The percentage recovery of $(R)$-enantiomer ranged from 97.1 to 100.1 for tablet dosage forms of pramipexole dihydrochloride monohydrate. In this study, the enantiomeric separation of pramipexole was achieved. The results demonstrated good linearity, precision, and accuracy for quantifying $(R)$-enantiomer in tablet dosage forms, suggesting the applicability of a quantitative method that uses polysaccharide-based CSPs in combination with organic-aqueous mobile phases.
\end{abstract}

Keywords Enantiomeric separation - Pramipexole - Dopamine agonist - Chiral HPLC method - Chiral stationary phase . Quantitative determination

\section{Introduction}

Pramipexole, a synthetic aminobenzothiazole with the chemical designation (6S)-6-N-propyl-4,5,6,7-tetrahydro1,3-benzothiazole-2,6-diamine [13], is a selective dopamine $D_{3}$ receptor agonist widely used as a symptomatic treatment for Parkinson's disease [4, 12]. It is a chiral compound with one chiral center that gives rise to two enantiomeric isomers, the $R$ - and $S$-form. Because the (S)-enantiomer has a much higher affinity to dopamine receptors than $(R)$-enantiomer [1], only $S$-form functions as pharmaceutically active pramipexole, whereas the inactive $(R)$-enantiomer is considered as an enantiomeric impurity that should be restricted.
Direct separation of pramipexole enantiomers has been implemented via liquid chromatographic method. Columns with polysaccharide-based chiral stationary phases (CSPs), such as Chiralpak AD and Chiralpak AD-H were applied using normal-phase separation in mixtures of alkanes (e.g., hexane) and low-molecular-weight alcohols $[10,14]$. Polysaccharide-based CSPs are materials of chromatographic column used in chiral HPLC which facilitate the enantiomeric resolution owing to their remarkable enantioselectivity combined with a broad versatility of solvents. The behavior of the column under normal phase and aqueous-organic mobile phase conditions has been extensively studied [3, 11]. Benzoate- or phenylcarbamate-derivatized polysaccharides are CSPs typically used in organic chemistry and pharmaceutical

Ching-Chia Chang, a10807@fda.gov.tw | ${ }^{1}$ Food and Drug Administration, Ministry of Health and Welfare, No. 161-2, Kunyang St, Nangang District, Taipei 11561, Taiwan.

SN Applied Sciences (2019) 1:50 | https://doi.org/10.1007/s42452-018-0057-0

Received: 8 July 2018 / Accepted: 12 November 2018 / Published online: 19 November 2018 
analysis [6]. Specifically, these CSPs were consisted of chiral-active cellulose and amylose, which the hydroxyl groups of the carbons at positions 2,3 , and 6 were substituted with benzoyl chlorides or phenyl isocyanates. In previous studies, it showed that the type and orientation of the phenyl substituents would affect the efficacy in the separation of enantiomeric isomer $[8,9]$. In addition, the selection of mobile phase mode will also affect the performance of column in enantioseparation which depends on the adaptability or advantage it brings about. While applying aqueous-organic phase mode in enantioseparation, numerous advantages could be obtained, such as shorter analysis time, favorable $\mathrm{S} / \mathrm{N}$ ratio, more environmentally friendly, higher efficiency and so on $[7,15]$. As a result, the aqueous-organic phase mode is a feasible and advantageous option in enantioseparation.

In this study, three different CSPs-Lux ${ }^{\circledR}$ Cellulose-1, Lux ${ }^{\circledast}$ Cellulose-3, and Lux ${ }^{\circledast}$ Cellulose- 4 were evaluated for their efficacy in separating enantiomeric isomers of pramipexole using an organic-aqueous mobile phase. Among three columns, Lux ${ }^{\circledast}$ Cellulose- 1 gave the best results. In addition, experiments of validation were carried out to verify the applicability of the new protocol in conducting the tests of enantiomeric impurity.

To our knowledge, the application of polysaccharidebased CSPs with organic-aqueous mobile phases has not been explored for the enantiomeric separation of pramipexole dihydrochloride monohydrate. In the study of enantioseparation, cellulose-based CSPs and an organicaqueous mobile phase were used. Additionally, the new HPLC protocol as a quality control method for the detection and quantification of $(R)$-enantiomeric impurities in pramipexole dihydrochloride monohydrate preparations was validated.

\section{Materials and methods}

\subsection{Chemicals and columns}

Reference standards of pramipexole (pramipexole dihydrochloride monohydrate) and (R)-enantiomer of pramipexole, $(R)$-pramipexole dihydrochloride, were purchased from the United States Pharmacopeia, USA, and Toronto Research Chemicals, Canada, respectively. Chemical structures of these two compounds are presented in Fig. 1. Tablets of pramipexole (label claim: $1 \mathrm{mg}$ pramipexole dihydrochloride monohydrate per tablet) were purchased from local supplier. HPLC-grade acetonitrile (ACN) was purchased from Merck, Germany. Ammonium bicarbonate was purchased from Acros Organics, Belgium. Diethylamine (DEA) was purchased from Sigma-Aldrich, USA. The analytical columns including

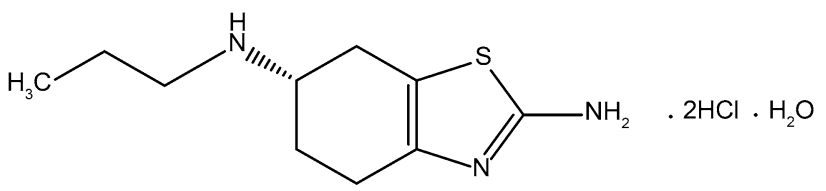

Pramipexole (pramipexole dihydrochloride monohydrate)

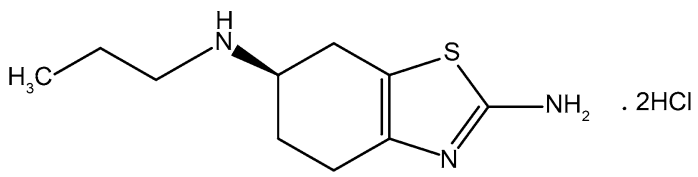

(R)-enantiomer of pramipexole

Fig. 1 Chemical structures of pramipexole enantiomers

Lux ${ }^{\circledast}$ Cellulose-1 [i.e. cellulose tris(3,5-dimethylphenylcarbamate)], Lux ${ }^{\circledR}$ Cellulose-3 [i.e. cellulose tris(4-methylbenzoate)], and Lux ${ }^{\circledast}$ Cellulose-4 [i.e. tris(4-chloro3-methylphenylcarbamate)]—were purchased from Phenomenex Inc., USA. The dimension of the columns was $250 \mathrm{~mm} \times 4.6 \mathrm{~mm}$, where the pore size was $5 \mu \mathrm{m}$.

\subsection{Apparatus and chromatographic conditions}

An HPLC system consisting of a Waters e2695 separation module combined with a Waters 2998 photodiode array detector (Waters Assoc., Milford, MA, USA) was utilized for method development and validation. The output signal was collected and processed using the Empower software. The HPLC protocol was optimized using the cellulose-based CSP column Lux ${ }^{\circledR}$ Cellulose- 1 at $25^{\circ} \mathrm{C}$ safeguarded by a $1-\mathrm{cm}$-long guard column. The flow rate was set at $1.0 \mathrm{~mL} \mathrm{~min}{ }^{-1}$. The chromatogram was collected at a wavelength of $254 \mathrm{~nm}$. Isocratic separation was conducted with the mobile phase consisting of solvent A (acetonitrile):solvent B ( $0.1 \%$ diethylamine with $20 \mathrm{mM}$ ammonium bicarbonate) $(30: 70, \mathrm{v} / \mathrm{v})$. The injection volume was $10 \mu \mathrm{L}$. In addition, cellulose-based CSP columns Lux ${ }^{\circledast}$ Cellulose- 3 and Lux ${ }^{\circledR}$ Cellulose- 4 were also used during preliminary testing for method development.

\subsection{Preparation of standard solutions}

Stock solutions of pramipexole $\left(3 \mathrm{mg} \mathrm{mL}^{-1}\right)$ and $(R)$ enantiomer $\left(300 \mu \mathrm{g} \mathrm{mL}^{-1}\right)$ were prepared by dissolving adequate amounts of the substances in ethanol. The analyte concentration of pramipexole was fixed as $300 \mu \mathrm{g} \mathrm{mL}^{-1}$. Working solutions of pramipexole and $(R)$ enantiomer were prepared using ethanol for dilution. 


\subsection{Preparation for tablet dosage solution}

Fourteen tablets were taken and grinded well into fine powder. The grinded power equivalent to amount of $3 \mathrm{mg}$ pramipexole dihydrochloride monohydrate was transferred to a $10 \mathrm{~mL}$ volumetric flask by adding $5 \mathrm{~mL}$ of ethanol. The sample was shaken for $5 \mathrm{~min}$ and then adequate ethanol was added to volume. Finally the sample solution was filtered through a $0.45 \mu \mathrm{m}$ filter prior to analysis.

\subsection{System suitability solution}

Solutions containing $10 \mu \mathrm{g} \mathrm{mL}^{-1}$ of pramipexole or $(R)$ enantiomer in ethanol were prepared.

\subsection{Method validation}

\subsubsection{Linearity of $(R)$-enantiomer of pramipexole}

Detector response linearity was assessed using different $(R)$-enantiomer solutions prepared by diluting with ethanol from stock solution for injecting into the HPLC. Different concentrations including $0.3,1.5,2.4,3.0$, and $3.6 \mu \mathrm{g} \mathrm{mL}^{-1}$ were used to assess the linear regression of the system. The regression curve was obtained by plotting the diagram of peak area versus the concentration by using least squares method. The RSD of the slope and Y-intercept of the calibration curve were calculated as well.

\subsubsection{Limit of detection (LOD) and limit of quantification (LOQ) for $(R)$-enantiomer of pramipexole}

The LOD is the lowest concentration of analyte that the signal can be detected above the baseline at the estimated signal-to-noise $(\mathrm{S} / \mathrm{N})$ ratio of 3 [5]. The $L O Q$ is the lowest concentration of analyte that can be quantified with suitable precision and accuracy at the estimated $\mathrm{S} / \mathrm{N}$ ratio of 10 [5]. The precision of the developed method was evaluated by analyzing six test solutions of $(R)$-enantiomer of pramipexole which was prepared at LOQ level and the relative standard deviation (RSD) of the peak area was calculated.

\subsubsection{Reproducibility of method}

To ensure the quality and reliability of the method, the reproducibility was evaluated by assessing repeatability and intermediate precision of peak areas for $(R)$-enantiomer of pramipexole.

The repeatability of the method was examined by analyzed with replicate injections $(\mathrm{n}=3)$ of the $(R)$-enantiomer solution at three concentrations, $0.3,2.4$ and $3.6 \mu \mathrm{g} \mathrm{mL}^{-1}$. The entire test included three successive injections on three consecutive days.

\subsubsection{Accuracy}

To verify the accuracy of the method, the reference standard was examined whereas there should be no $(R)$-enantiomer detected in the tablet dosage forms sample. Standard addition and recovery experiments were conducted to determine the accuracy of the method in quantifying $(R)$-enantiomer in tablet dosage form samples.

The experiment was evaluated by assaying freshly prepared solutions in triplicate at three concentrations, 0.1, 0.8 , and $1.2 \%$ (equivalent to the concentration of $0.3,2.4$, and $3.6 \mu \mathrm{g} \mathrm{mL}^{-1}$ ) of the target analyte pramipexole dihydrochloride monohydrate. The recovery of $(R)$-enantiomer was derived from the slope and Y-intercept of the calibration curve obtained in linearity and recovery experiment.

\section{Results and discussion}

\subsection{Method development and optimization}

In previous studies, enantiomeric separations on polysaccharide-based CSP columns were mostly conducted in normal phase mode using alkane solutions with lowmolecular-weight alcohols as eluents. However, the usage of these columns under different mobile phase mode, such as aqueous-organic eluents, was still awaited to explore. In this study, it was aimed to broaden the application of the column consisting of polysaccharide-based CSPs in separation of the enantiomers. The optimized method demonstrated good linearity, precision, and accuracy for quantifying the target enantiomer. In addition to these advantages, several goals were proposed for this study:

(a) To develop an analytical method using CSPs in aqueous-organic mobile phase mode.

(b) To reduce the potential risk for intrinsic instrument errors caused by frequent changes between eluents used in the normal mobile phase and the aqueousorganic mobile phase mode.

(c) To meet the requirements for enantiomeric purity specified in the pharmacopeia.

The racemates investigated in this study were typically analyzed using CSPs with "normal phase" eluents. Three different polysaccharide-based columns, Lux ${ }^{\circledast}$ Cellulose-1, Lux ${ }^{\circledast}$ Cellulose-3 and Lux ${ }^{\circledast}$ Cellulose-4 (Fig. 2) were applied with aqueous-organic eluents for the experiments. Good separation was achieved for the racemates of pramipexole. Further experiments were performed to optimize the separation efficiency of pramipexole enantiomers.

Three CSP columns were used in optimizing the variables of the method including column temperature and the 
Fig. 2 Chemical structures of CSPs<smiles>Cc1cc(C)cc(NC(=O)OCC[C@H](OC(=O)Nc2cc(C)cc(C)c2)[C@H](COC(=O)Nc2cc(C)cc(C)c2)C(C)(C)C)c1</smiles>

Lux® Cellulose-1 Cellulose tris (3, 5-dimethylphenylcarbamate)<smiles>Cc1cc(NC(=O)OCC[C@H](OC(=O)Nc2ccc(Cl)c(C)c2)[C@H](OC(=O)Nc2ccc(Cl)c(C)c2)C(C)(C)C)ccc1Cl</smiles>

Lux® Cellulose-4

Cellulose tris (4-chloro-3-methylphenylcarbamate)<smiles>Cc1ccc(C(=O)OCC[C@H](OC(=O)c2ccc(C)cc2)[C@@H](OC(=O)c2ccc(C)cc2)C(C)(C)C)cc1</smiles>

Lux® Cellulose-3

Cellulose tris (4-methylbenzoate)

content of organic modifier in the eluent. The parameters of analysis were as follows-mobile phase consisting of solvent A (acetonitrile):solvent B ( $0.1 \%$ diethylamine with $20 \mathrm{mM}$ ). In the pretest of column performance, inadequate separation was observed while applying Lux ${ }^{\circledast}$ Cellulose-3 and Lux ${ }^{\circledR}$ Cellulose-4 (Fig. 3). On contrary, an improvement in resolution was observed when applying Lux ${ }^{\circledR} \mathrm{Cel}-$ lulose-1 column under consistent conditions. The results referred to the essential effects on enantioseparation according to the type of column. It has been discussed in the literatures that the performance in enantioseparation varies with different CSP, i.e. polysaccharide linkage. The enantioseparation was affected by the mechanism of inner retention between CSP and analyte resulted from various possible bonding mechanism and interactions. Among those mechanisms, the steric effect from chiral groove is the key role in the chiral recognition [16].

To improve the efficiency for a broad application of the method, the conditions of analysis were optimized using Lux ${ }^{\circledast}$ Cellulose-1 column to reduce the processing time while the adequate enantioseparation was still retained. Typically, the increase in the proportion of organic solvent with mobile phase reduces the retention time of the target compound, whereas the decrease of column temperature 
Fig. 3 Separation of pramipexole racemate in aqueousorganic mobile phase on three different polysaccharide-based CSP columns: a Lux ${ }^{\circledast}$ Cellulose-1; b Lux ${ }^{\circledast}$ Cellulose-4; c Lux ${ }^{\oplus}$ Cellulose-3. Conditions of analysis were as follow: mobile phase consisting of solvent $A$ (acetonitrile):solvent $\mathrm{B}(0.1 \%$ diethylamine with $20 \mathrm{mM}$ ammonium bicarbonate) $(20: 80, v / v)$, flow rate

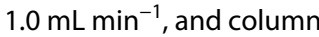
temperature $35^{\circ} \mathrm{C}$
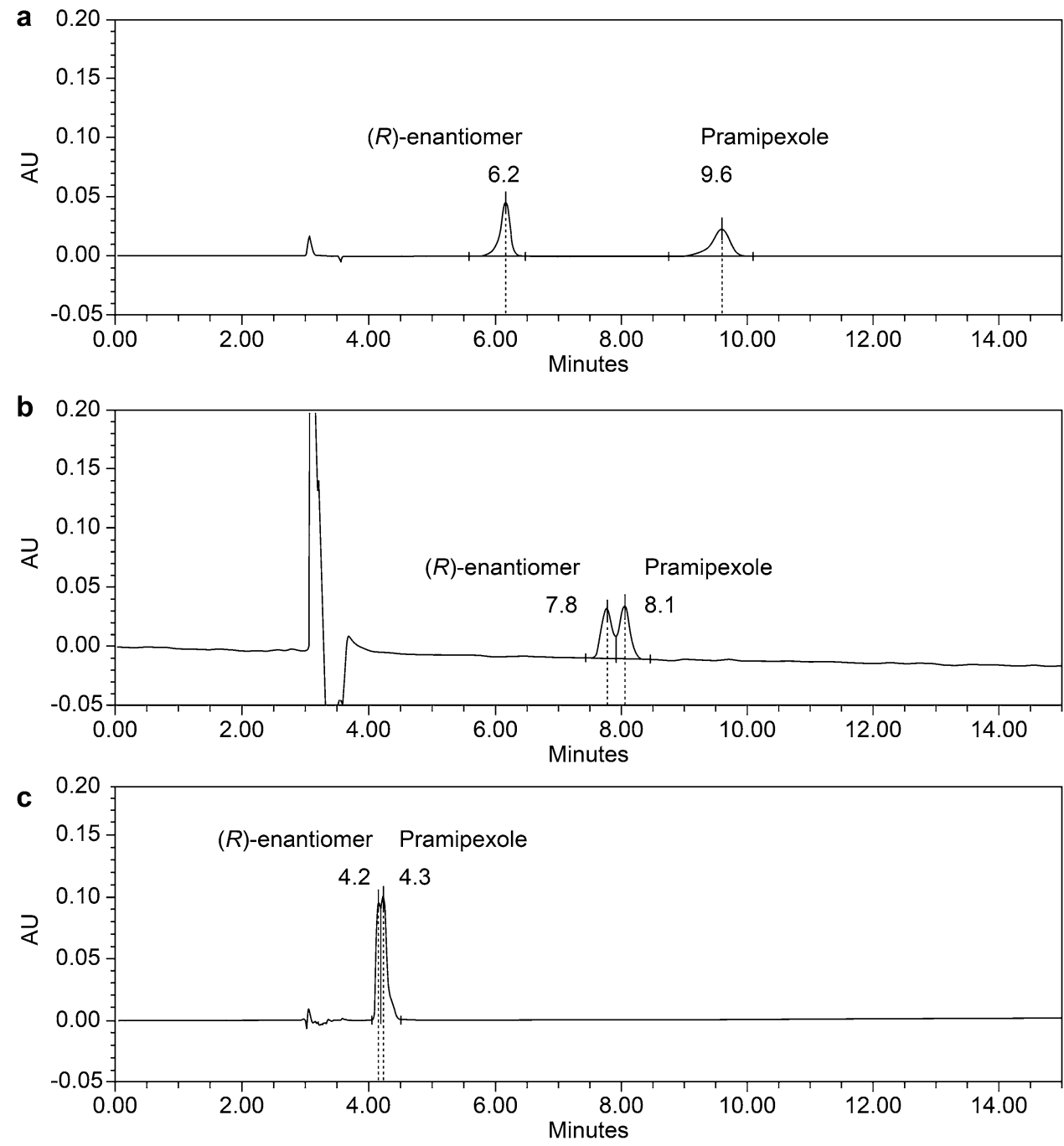

improves the resolution between the enantiomers. For the effect of temperature, though it was found that higher column temperature resulted in better enantioseparation in the literatures [2, 15]; however, it demonstrated a quite different trend in this study. Experiments were carried out to verify the effect of column temperature. Under consistent parameters of elution, it showed that the resolution between $(R)$-enantiomer and pramipexole increased with the decrease of column temperature (Fig. 4). On the other hand, to examine the effects of the ACN content, experiments were performed. From the results, although the difference of retention time for both compounds would increase with the decrease of ACN content; however, there would be more impacts on retention factor $(\mathrm{K})$ and resolution $\left(R_{s}\right)$ which leads to the increase of total analysis time and the decrease of peak height (Fig. 5). In sake of the performance and sensitivity of the analyzing method, the ACN content of $30 \%$ was adapted. In the optimization experiment, with a proper ACN proportion and a lower column temperature, a good separation of the enantiomers was achieved.

In a recent study by Zhang et al., the authors observed that a basic solution of ammonium bicarbonate adjusted at $\mathrm{pH} 9.0$ was the most efficient aqueous mobile phase component to improve the resolution of basic enantiomers [17]. The target compounds in this study are also basic. Therefore, $0.1 \%$ DEA was added to the mobile phase to improve the peak shapes, and thus, facilitate the column performance in separating the enantiomers (Resolution, $R_{s}>8.0$ ). The final conditions in the protocol were as follows-mobile phase consisting of solvent $A$ (acetonitrile):solvent $B(0.1 \%$ diethylamine with $20 \mathrm{mM}$ ammonium bicarbonate) (30:70, v/v), a flow rate of $1.0 \mathrm{~mL} \mathrm{~min} \mathrm{~m}^{-1}$, and a column temperature of $25^{\circ} \mathrm{C}$. The results of the method optimization were shown in Fig. 6. Importantly, the retention times for $(R)$-enantiomer and pramipexole were 4.3 and 6.2 min, respectively. A summary of the system suitability tests using chiral HPLC 
Fig. 4 Effect of column temperature on resolution $\left(\mathrm{R}_{\mathrm{s}}\right)$, and retention time $(\mathrm{min})$ on Lux ${ }^{\oplus}$ Cellulose- 1 column in aqueous-organic mobile phase eluent. Conditions of analysis were as follow: mobile phase consisting of solvent A (acetonitrile):solvent $\mathrm{B}(0.1 \%$ diethylamine with $20 \mathrm{mM}$ ammonium bicarbonate) $(30: 70, v / v)$, flow rate $1.0 \mathrm{~mL} \mathrm{~min}^{-1}$

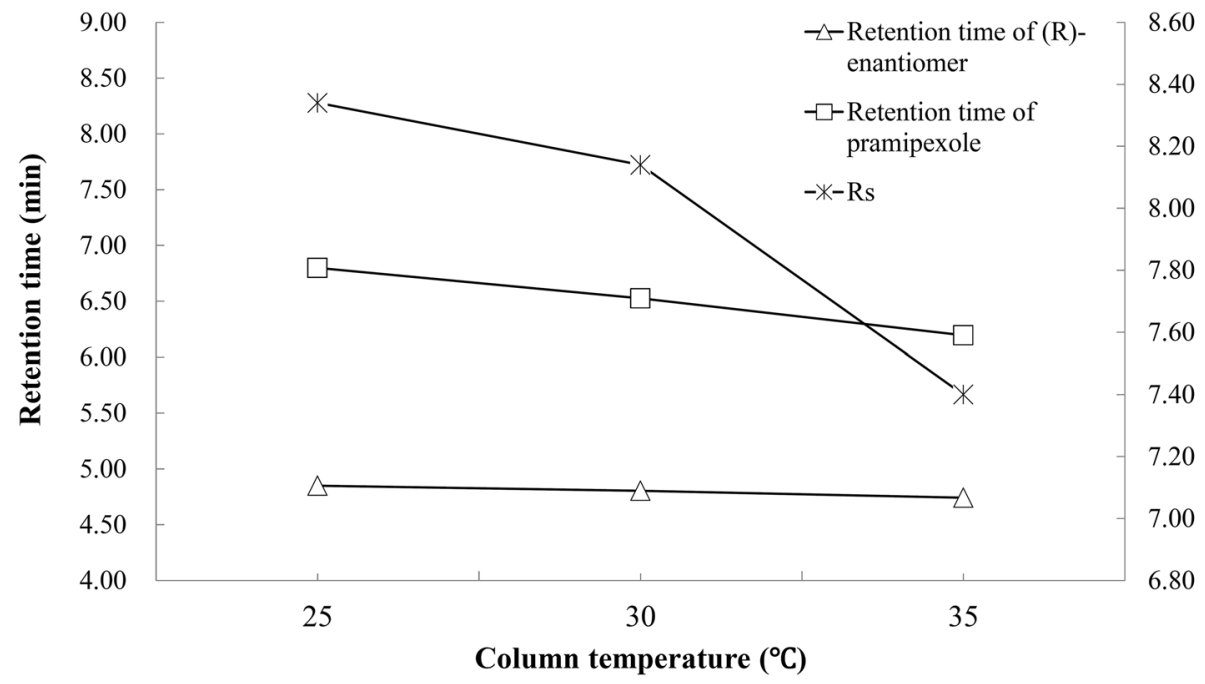

method on a Lux ${ }^{\circledast}$ Cellulose-1 column was provided in Table 1.

\subsection{Results of method validation}

Good linearity was achieved for the $(R)$-enantiomer of pramipexole over a concentration range of $0.3-3.6 \mu \mathrm{g} \mathrm{mL}^{-1}$ with a correlation coefficient of $\mathrm{R}=0.9999$ derived from the linear regression equation $y=11,287.1835 x-258.8004$ (Table 2). The results indicated that the peak area of the analyte was strongly correlated with the concentration.

During the reproducibility tests of the method, the RSD values of injection repeatability for the $(R)$-enantiomer of pramipexole were $2.64,1.28$, and 1.72 , respectively. Results of intermediate precision showed that the RSD values were $1.54,2.20$, and 1.85 , respectively (Table 2 ).

The estimated LOD and LOQ values for $(R)$-enantiomer of pramipexole were 0.1 (at S/N ratio of 3) and $0.3 \mu \mathrm{g} \mathrm{mL}^{-1}$ (at $S / N$ ratios of 10 ), respectively. The precision of the method for $(R)$-enantiomer quantification in pramipexole preparations at LOQ had an RSD value of $5.48 \%$ (Table 2).

The recovery of $(R)$-enantiomer was assessed at concentration $0.1,0.8$, and $1.2 \%$ of target analyte pramipexole dihydrochloride monohydrate. The estimated recovery (\%) ranged between 97.1 and 100.1, derived from the slope and $\mathrm{Y}$-intercept of the calibration curve from the linear regression analysis (Table 3 ).

Fig. 5 Effect of acetonitrile content (\%) on a resolution $\left(R_{s}\right)$ and retention factor $(k)$ of $(R)$-enantiomer, and $\mathbf{b}$ retention time $(\mathrm{min})$ in aqueous-organic mobile phase eluent on Lux ${ }^{\circledast}$ Cellulose-1 column. Conditions of analysis were as follow: mobile phase consisting of solvent A (acetonitrile):solvent B (0.1\% diethylamine with $20 \mathrm{mM}$ ammonium bicarbonate), flow rate $1.0 \mathrm{~mL} \mathrm{~min}^{-1}$, and column temperature $25^{\circ} \mathrm{C}$

\section{Conclusion}

A rapid, simple, and feasible chiral HPLC method using an aqueous-organic mobile phase in isocratic mode was developed for the separation of $(R)$-enantiomer from pramipexole. Cellulose-based chiral column Lux Cellulose-1 column was approved for the enantioseparation 


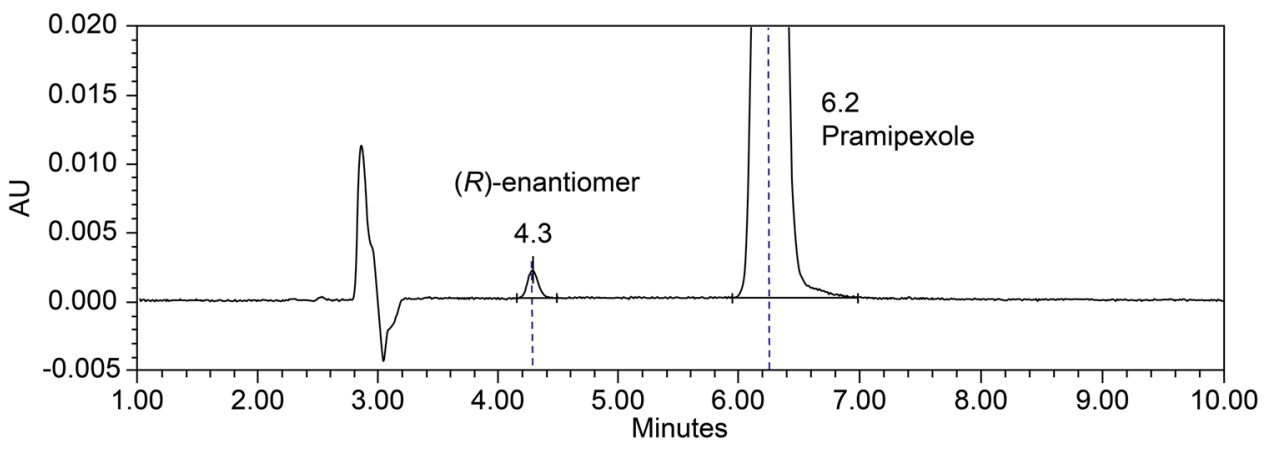

Fig. 6 Optimized conditions for separation of $(R)$-enantiomer $\left(1.2 \mu \mathrm{g} \mathrm{mL} \mathrm{m}^{-1}\right)$ from pramipexole $\left(300 \mu \mathrm{g} \mathrm{mL}^{-1}\right)$ on Lux Cellulose-1 column in aqueous-organic mobile phase eluent. Conditions of analysis were as follow: mobile phase consisting of solvent $A$

Table 1 System suitability report

\begin{tabular}{lllrl}
\hline Compound & $R_{\mathrm{t}}(\mathrm{min})$ & $R_{\mathrm{s}}$ & $N$ & \multicolumn{1}{l}{$T$} \\
\hline Pramipexole & 6.5 & & 9678 & 1.06 \\
$(R)$-enantiomer & 4.6 & 9.2 & 11,418 & 1.03 \\
\hline
\end{tabular}

Determinations: $\mathrm{n}=3 ; R_{t}$ retention time, $R_{\mathrm{s}}$ resolution, $N$ USP plate count, $T$ USP tailing factor

Table 2 Method validation for $(R)$-enantiomer of pramipexole

\begin{tabular}{|c|c|}
\hline Validation parameter & Results \\
\hline \multicolumn{2}{|l|}{ Repeatability $(n=3, \%$ RSD) } \\
\hline Peak area $\left(0.3 \mu \mathrm{g} \mathrm{mL}^{-1}\right)$ & 2.64 \\
\hline Peak area $\left(2.4 \mu \mathrm{g} \mathrm{mL}^{-1}\right)$ & 1.28 \\
\hline Peak area $\left(3.6 \mu \mathrm{g} \mathrm{mL}^{-1}\right)$ & 1.72 \\
\hline \multicolumn{2}{|c|}{ Intermediate precision ( $n=9, \% R S D)$} \\
\hline Peak area $\left(0.3 \mu \mathrm{g} \mathrm{mL}^{-1}\right)$ & 1.54 \\
\hline Peak area $\left(2.4 \mu \mathrm{g} \mathrm{mL}^{-1}\right)$ & 2.20 \\
\hline Peak area $\left(3.6 \mu \mathrm{g} \mathrm{mL}^{-1}\right)$ & 1.85 \\
\hline \multicolumn{2}{|l|}{ LOD-LOQ } \\
\hline $\operatorname{LOD}\left(\mu \mathrm{g} \mathrm{mL}^{-1}\right)$ & 0.1 \\
\hline $\mathrm{LOQ}\left(\mu \mathrm{g} \mathrm{mL}^{-1}\right)$ & 0.3 \\
\hline Precision at LOQ (RSD in \%) & 5.48 \\
\hline \multicolumn{2}{|l|}{ Linearity } \\
\hline Calibration range $\left(\mu \mathrm{g} \mathrm{mL}^{-1}\right)$ & $0.3-3.6$ \\
\hline Calibration points & 5 \\
\hline Correlation coefficient & 0.9999 \\
\hline Slope & $11,287.1835$ \\
\hline Intercept & -258.8004 \\
\hline
\end{tabular}

of pramipexole with aqueous-organic mobile phase eluent consisting of solvent $A$ (acetonitrile):solvent B $(0.1 \%$ diethylamine with $20 \mathrm{mM}$ ammonium bicarbonate) (30:70, $\mathrm{v} / \mathrm{v}$ ). The elution time was about $7.0 \mathrm{~min}$ and the resolution between the enantiomers was greater than 8.0. The linear (acetonitrile):solvent B ( $0.1 \%$ diethylamine with $20 \mathrm{mM}$ ammonium bicarbonate) $(30: 70, \mathrm{v} / \mathrm{v})$, flow rate $1.0 \mathrm{~mL} \mathrm{~min}^{-1}$, and column temperature $25^{\circ} \mathrm{C}$

Table 3 Recovery of $(R)$ enantiomer

\begin{tabular}{lrr}
\hline $\begin{array}{l}\text { Added } \\
(\mu \mathrm{g}) \\
(\mathrm{n}=3)\end{array}$ & \% Recovery & \% RSD \\
\hline 0.3 & 97.1 & 1.86 \\
2.4 & 100.1 & 1.56 \\
3.6 & 100.0 & 0.51 \\
\hline
\end{tabular}

range of the method was $0.3-3.6 \mu \mathrm{g} \mathrm{mL}^{-1}$ with LOD and LOQ for $(R)$-enantiomer were $0.1 \mu \mathrm{g} \mathrm{mL} L^{-1}$ and $0.3 \mu \mathrm{g} \mathrm{mL}^{-1}$, respectively. The estimated recovery $(\%)$ of $(R)$-enantiomer ranged between 97.1 and 100.1 at concentrations of 0.1 , 0.8 , and $1.2 \%$. The established method showed satisfactory data for all validation parameters. The enantiomeric separation of pramipexole was quantitative. It demonstrated good linearity, precision, and accuracy for the quantification of $(R)$-enantiomer in tablet dosage forms, which put the usage of polysaccharide-based CSPs in combination with organic-aqueous mobile phases for quantitative determinations into practice.

Acknowledgements The authors are grateful to the Food and Drug Administration (Taipei, Taiwan) for the financial support of this work.

Funding This study was funded by Food and Drug Administration (Taipei, Taiwan) (Grant Number MOHW106-FDA-D-315-000738).

\section{Compliance with ethical standards}

Conflict of interest The authors declare that they have no conflict of interest.

\section{References}

1. Chang WI, Weber M, Breier MR, Saint Marie RL, Hines SR, Swerdlow NR (2012) Stereochemical and neuroanatomical selectivity 
of pramipexole effects on sensorimotor gating in rats. Brain Res 1437:69-76

2. Foroughbakhshfasaei M, Szabó ZI, Tóth G (2018) Validated LC method for determination of enantiomeric purity of apremilast using polysaccharide-type stationary phases in polar organic mode. Chromatographia. https://doi.org/10.1007/s1033 7-018-3546-9

3. Franco P, Zhang T (2008) Common approaches for efficient method development with immobilised polysaccharide-derived chiral stationary phases. J Chromatogr B Anal Technol Biomed Life Sci 875(1):48-56

4. Hametner EM, Seppi K, Poewe W (2012) Role and clinical utility of pramipexole extended release in the treatment of early Parkinson's disease. Clin Interv Aging 7:83-88

5. ICH (2005) Q2(R1) validation of analytical procedures: text and methodology. In: international conference on harmonisation of technical requirements for registration of pharmaceuticals for human use. ICH home page. http://www.ich.org. Accessed 1 Feb 2018

6. Liu RQ, Bai LY, Zhang YJ, Zhang YP (2013) Green synthesis of a typical chiral stationary phase of cellulose-tris(3, 5-dimethylphenylcarbamate). Chem Cent J 7(1):129

7. Matarashvili I, Shvangiradze I, Chankvetadze L, Sidamonidze S, Takaishvili N, Farkas T, Chankvetadze B (2015) High-performance liquid chromatographic separations of stereoisomers of chiral basic agrochemicals with polysaccharide-based chiral columns and polar organic mobile phases. J Sep Sci 38(24):4173-4179

8. Okamoto Y, Kawashima M, Hatada K (1986) Chromatographic resolution: $\mathrm{XI}$. Controlled chiral recognition of cellulose triphenylcarbamate derivatives supported on silica gel. J Chromatogr A 363(2):173-186

9. Okamoto Y, Aburatani R, Hatada K (1987) Chromatographic chiral resolution: XIV. Cellulose tribenzoate derivatives as chiral stationary phases for high-performance liquid chromatography. J Chromatogr A 389(1):95-102

10. Pathare DB, Jadhav AS, Shingare MS (2006) Validated chiral liquid chromatographic method for the enantiomeric separation of pramipexole dihydrochloride monohydrate. J Pharm Biomed Anal 41(4):1152-1156

11. Peng L, Jayapalan S, Chankvetadze B, Farkas T (2010) Reversedphase chiral HPLC and LC/MS analysis with tris(chloromethylp henylcarbamate) derivatives of cellulose and amylose as chiral stationary phases. J Chromatogr A 1217(44):6942-6955

12. Piercey MF (1998) Pharmacology of pramipexole, a dopamine $\mathrm{D}_{3}$-preferring agonist useful in treating Parkinson's disease. Clin Neuropharmacol 21(3):141-151

13. PubChem Compound Database (2004) US National Institutes of Health. CID $=119570$. https://pubchem.ncbi.nlm.nih.gov/compo und/119570. Accessed 27 Feb 2018

14. Ramisetti NR, Kuntamukkala R, Arnipalli MS (2015) Development of a validated LC method for separation of process-related impurities including the $R$-enantiomer of $S$-pramipexole on polysaccharide chiral stationary phases. Chirality 27(7):430-435

15. Szabó ZI, Foroughbakhshfasaei M, Gál R, Horváth $P$, Komjáti $B$, Noszál B, Tóth G (2018) Chiral separation of lenalidomide by liquid chromatography on polysaccharide-type stationary phases and by capillary electrophoresis using cyclodextrin selectors. J Sep Sci 41(6):1414-1423

16. Yashima E (2001) Polysaccharide-based chiral stationary phases for high-performance liquid chromatographic enantioseparation. J Chromatogr A 906(1-2):105-125

17. Zhang T, Nguyen D, Franco P (2010) Reversed-phase screening strategies for liquid chromatography on polysaccharide-derived chiral stationary phases. J Chromatogr A 1217(7):1048-1055 